\title{
ON THE LINEARIZED STABILITY OF AGE-STRUCTURED MULTISPECIES POPULATIONS
}

\author{
JOZSEF Z. FARKAS
}

Received 3 February 2006; Revised 24 April 2006; Accepted 8 May 2006

We consider a general nonlinear age-structured population model with $n$ interacting species. We deduce the characteristic function in the form of a determinant of an $n$-by$n$ matrix. Then we formulate some biologically meaningful sufficient conditions for the stability (resp., instability) of positive stationary solutions of the system.

Copyright (c) 2006 Jozsef Z. Farkas. This is an open access article distributed under the Creative Commons Attribution License, which permits unrestricted use, distribution, and reproduction in any medium, provided the original work is properly cited.

\section{Introduction}

In [13] Prüß investigated a very general nonlinear age-structured model consisting of $n$ species. He proved the principle of linearized stability, which means that the asymptotic behavior of stationary solutions of the nonlinear system is determined by the spectrum of the linearized operator. In other words the stability is determined by the roots of a complex valued characteristic function as it is claimed in [2] for general physiologically structured population models, as well. More recently Kato [11] proved the principle of linearized stability for more general abstract nonlinear evolution equations of type $(d / d t) u(t)+A u(t)=0$ where $A$ is a quasi $m$-accretive operator.

In the present paper, we extend the approach first used in [5] then later in [6-8], to actually deduce the characteristic function and show that it can be obtained as a determinant of an $n$-by- $n$ matrix as claimed in [2]. We restrict ourselve to the frequently studied case where the vital rate functions depend on the total population quantity, but it should be clear that the method can be extended to more general cases of multispecies structured population models.

In [13], Prüß discussed the stability of stationary solutions with trivial components and established stability conditions for a very special two-species system. He also derived conditions for the positivity of the governing linear semigroup and he used it to prove an instability result. 
2 Stability of an age-structured multispecies model

In the case when the linearized system is governed by a positive semigroup one has that the spectral bound of the operator is real and belongs to the spectrum (see, e.g., [4]). In other words, there is a strictly dominant real root of the corresponding characteristic function.

Prüß and others in the literature used the positivity of the governing semigroup to prove instability results. This might be natural since instability often occurs in the case of a dominant real root. On the other hand one can prove the instability results without imposing the technical and rather restrictive positivity conditions, as we will see later, and use the positivity conditions to prove stability results. The main advantage of the positivity is that we can restrict ourselves to real calculus when addressing stability questions.

In fact, using the characteristic function we are able to prove a more general instability result, compared to the one in [13], without imposing any of the positivity conditions. Then we establish a stability criterion under rather restrictive conditions which impose the positivity conditions given in [13].

We mention here the works $[1,3,9,10,12,14]$ and the references therein for developments in structured population dynamics.

Consider the system describing the dynamics of an age-structured population with $n$ interacting species,

$$
\begin{gathered}
p_{a}^{i}(a, t)+p_{t}^{i}(a, t)=-\mu^{i}(a, \mathbf{P}) p^{i}(a, t), \\
p^{i}(0, t)=\int_{0}^{\infty} \beta^{i}(a, \mathbf{P}) p^{i}(a, t) d a, \quad \mathbf{P}=\left(P^{1}, \ldots, P^{n}\right), P^{i}=\int_{0}^{\infty} p^{i}(a, t) d a, \\
p^{i}(a, 0)=p_{0}^{i}(a), \quad a, t \geq 0, i=1, \ldots, n,
\end{gathered}
$$

with sufficiently smooth (see [13]) vital rate functions $\beta^{i}, \mu^{i} \geq 0, i=1, \ldots, n$.

Stationary solutions $\left(p_{*}^{1}(a), \ldots, p_{*}^{n}(a)\right)$ of the system $(1.1)$ can be obtained as (this is a standard calculation, see, e.g., [5])

$$
\begin{aligned}
p_{*}^{i}(a) & =\frac{P_{*}^{i} \exp \left\{-\int_{0}^{a} \mu^{i}\left(s, \mathbf{P}_{*}\right) d s\right\}}{\int_{0}^{\infty} \exp \left\{-\int_{0}^{a} \mu^{i}\left(s, \mathbf{P}_{*}\right) d s\right\} d a}=: \frac{P_{*}^{i} \pi^{i}\left(a, \mathbf{P}_{*}\right)}{\int_{0}^{\infty} \pi^{i}\left(a, \mathbf{P}_{*}\right) d a} \\
& =p_{*}^{i}(0) \pi^{i}\left(a, \mathbf{P}_{*}\right), \quad i=1, \ldots, n,
\end{aligned}
$$

where $\mathbf{P}_{*}=\left(P_{*}^{1}, \ldots, P_{*}^{n}\right)$ is the solution of the equations

$$
1=R^{i}(\mathbf{P})=\int_{0}^{\infty} \beta^{i}(a, \mathbf{P}) \pi^{i}(a, \mathbf{P}) d a, \quad i=1, \ldots, n .
$$

Here $R^{i}$ denotes the so-called net reproduction function, the expected number of newborns to be produced by an individual. Existence of nonnegative stationary solutions (of a more general system) is discussed in detail in [13]. 


\section{The linearized system and stability}

We introduce the variation $u^{i}(a, t)=p^{i}(a, t)-p_{*}^{i}(a), i=1, \ldots, n$, in $(1.1)$ and use the approximations

$$
\begin{aligned}
& \mu^{i}(a, \mathbf{P})=\mu^{i}\left(a, \mathbf{P}_{*}\right)+\sum_{j=1}^{n} \mu_{P_{j}}^{i}\left(a, \mathbf{P}_{*}\right)\left(P^{j}-P_{*}^{j}\right)+\text { higher-order terms, } i=1, \ldots, n, \\
& \beta^{i}(a, \mathbf{P})=\beta^{i}\left(a, \mathbf{P}_{*}\right)+\sum_{j=1}^{n} \beta_{P_{j}}^{i}\left(a, \mathbf{P}_{*}\right)\left(P^{j}-P_{*}^{j}\right)+\text { higher-order terms, } \quad i=1, \ldots, n,
\end{aligned}
$$

to obtain the linear system

$$
\begin{gathered}
u^{i}(a, t)_{a}+u^{i}(a, t)_{t}=-\mu^{i}\left(a, \mathbf{P}_{*}\right) u^{i}(a, t)-p_{*}^{i}(a)\left(\sum_{j=1}^{n} \mu_{P_{j}}^{i}\left(a, \mathbf{P}_{*}\right) \int_{0}^{\infty} u^{j}(a, t) d a\right), \\
u^{i}(0, t)=\int_{0}^{\infty} \beta^{i}\left(a, \mathbf{P}_{*}\right) u^{i}(a, t)+p_{*}^{i}(a)\left(\sum_{j=1}^{n} \beta_{P_{j}}^{i}\left(a, \mathbf{P}_{*}\right) \int_{0}^{\infty} u^{j}(a, t) d a\right) d a, \quad i=1, \ldots, n .
\end{gathered}
$$

Now substituting $u^{i}(a, t)=e^{\lambda t} U^{i}(a), i=1, \ldots, n$, into the linearized system (2.2) and making use of the notation $\bar{U}^{j}=\int_{0}^{\infty} U^{j}(a) d a, j=1, \ldots, n$, we obtain

$$
\begin{gathered}
U^{i}(a)^{\prime}+U^{i}(a)\left(\lambda+\mu^{i}\left(a, \mathbf{P}_{*}\right)\right)=-p_{*}^{i}(a)\left(\sum_{j=1}^{n} \mu_{P_{j}}^{i}\left(a, \mathbf{P}_{*}\right) \bar{U}^{j}\right), \\
U^{i}(0)=\int_{0}^{\infty} \beta^{i}\left(a, \mathbf{P}_{*}\right) U^{i}(a)+p_{*}^{i}(a)\left(\sum_{j=1}^{n} \beta_{P_{j}}^{i}\left(a, \mathbf{P}_{*}\right) \bar{U}^{j}\right) d a, \quad i=1, \ldots, n .
\end{gathered}
$$

Using the relation (1.2), the solution of (2.3)-(2.4) can be written as

$$
U^{i}(a)=e^{-\lambda a} \pi^{i}\left(a, \mathbf{P}_{*}\right)\left(U^{i}(0)-p_{*}^{i}(0) \int_{0}^{a} e^{\lambda s} \sum_{j=1}^{n} \mu_{P_{j}}^{i}\left(s, \mathbf{P}_{*}\right) \bar{U}^{j} d s\right), \quad i=1, \ldots, n .
$$

Integration of (2.5) yields

$$
\bar{U}^{i}=A^{1, i}(\lambda) U^{i}(0)+\sum_{j=1}^{n} A_{j}^{i}(\lambda) \bar{U}^{j}, \quad i=1, \ldots, n,
$$

with

$$
\begin{gathered}
A^{1, i}(\lambda)=\int_{0}^{\infty} e^{-\lambda a} \pi^{i}\left(a, \mathbf{P}_{*}\right) d a, \quad i=1, \ldots, n, \\
A_{j}^{i}(\lambda)=-p_{*}^{i}(0) \int_{0}^{\infty} e^{-\lambda a} \pi^{i}\left(a, \mathbf{P}_{*}\right) \int_{0}^{a} e^{\lambda s} \mu_{P_{j}}^{i}\left(s, \mathbf{P}_{*}\right) d s d a, \quad i, j=1, \ldots, n,
\end{gathered}
$$


4 Stability of an age-structured multispecies model

while substituting (2.5) into (2.4) yields

$$
U^{i}(0)=A^{2, i}(\lambda) U^{i}(0)+\sum_{j=1}^{n} \bar{A}_{j}^{i}(\lambda) \bar{U}^{j}, \quad i=1, \ldots, n,
$$

where

$$
\begin{gathered}
A^{2, i}(\lambda)=\int_{0}^{\infty} e^{-\lambda a} \beta^{i}\left(a, \mathbf{P}_{*}\right) \pi^{i}\left(a, \mathbf{P}_{*}\right) d a, \quad i=1, \ldots, n, \\
\bar{A}_{j}^{i}(\lambda)=p_{*}^{i}(0) \int_{0}^{\infty} \beta_{P_{j}}^{i}\left(a, \mathbf{P}_{*}\right) \pi^{i}\left(a, \mathbf{P}_{*}\right), \\
-e^{-\lambda a} \pi^{i}\left(a, \mathbf{P}_{*}\right) \beta^{i}\left(a, \mathbf{P}_{*}\right) \int_{0}^{a} e^{\lambda s} \mu_{P_{j}}^{i}\left(s, \mathbf{P}_{*}\right) d s d a, \quad i, j=1, \ldots, n .
\end{gathered}
$$

Rewrite the linear system (2.6)-(2.8) in the form $\mathbf{K}(\lambda) \mathbf{U}=\mathbf{0}$, where $\mathbf{U}=\left(U^{1}(0), \ldots, U^{n}(0)\right.$, $\left.\bar{U}^{1}, \ldots, \bar{U}^{n}\right)^{T}, \mathbf{0}=(0, \ldots, 0)^{T}$ and the $2 n$-by- $2 n$ matrix $\mathbf{K}(\lambda)$ is as follows:

$$
\mathbf{K}(\lambda)=\left(\begin{array}{ll}
\mathbf{M}_{1}(\lambda) & \mathbf{M}_{2}(\lambda) \\
\mathbf{M}_{3}(\lambda) & \mathbf{M}_{4}(\lambda)
\end{array}\right)
$$

where

$$
\begin{gathered}
\mathbf{M}_{1}(\lambda)=\operatorname{diag}\left(A^{1,1}(\lambda), \ldots, A^{1, n}(\lambda)\right), \\
\mathbf{M}_{3}(\lambda)=\operatorname{diag}\left(\left(A^{2,1}(\lambda)-1\right), \ldots,\left(A^{2, n}(\lambda)-1\right)\right)
\end{gathered}
$$

are $n$-by- $n$ diagonal matrices, while

$$
\begin{gathered}
\mathbf{M}_{2}(\lambda)_{(i \cdot j)}= \begin{cases}A_{j}^{i}(\lambda) & \text { if } i \neq j, i, j=1, \ldots, n, \\
A_{i}^{i}(\lambda)-1 & \text { if } i=j, i=1, \ldots, n,\end{cases} \\
\mathbf{M}_{4}(\lambda)_{(i, j)}=\left\{\bar{A}_{j}^{i}(\lambda), i, j=1, \ldots, n\right\} .
\end{gathered}
$$

Thus [13, Theorem 2] takes the following form.

THeOREM 2.1. The stationary solution $\left(p_{*}^{1}(a), \ldots, p_{*}^{n}(a)\right)$ is stable if all the roots of the (continuous) function $\operatorname{det}(\mathbf{K}(\lambda))=|\mathbf{K}(\lambda)|: \mathbf{C} \rightarrow \mathbf{C}$ are in the left-hand plane and it is unstable if there is a root with positive real part.

In the following, we treat the stability of (strictly) positive stationary solutions that is $P_{*}^{i}>0, i=1, \ldots, n$.

Theorem 2.2. One has

$$
\lim _{\lambda \rightarrow \infty}|\mathbf{K}(\lambda)|=-1^{n}
$$

the limit being taken in $\mathbf{R}$. 
Proof. Straightforward calculations show that

$$
\lim _{\lambda \rightarrow \infty} A^{1, i}(\lambda)=\lim _{\lambda \rightarrow \infty} A^{2, i}(\lambda)=\lim _{\lambda \rightarrow \infty} A_{j}^{i}(\lambda)=0, \quad i, j=1, \ldots, n,
$$

which means that

$$
\lim _{\lambda \rightarrow \infty} \mathbf{M}_{1}(\lambda)=\mathbf{0}, \quad \lim _{\lambda \rightarrow \infty} \mathbf{M}_{2}(\lambda)=\lim _{\lambda \rightarrow \infty} \mathbf{M}_{3}(\lambda)=-\mathbf{I}_{n}
$$

that is,

$$
\lim _{\lambda \rightarrow \infty} \mathbf{K}(\lambda)=\left(\begin{array}{cc}
\mathbf{0} & -\mathbf{I}_{n} \\
-\mathbf{I}_{n} & \overline{\mathbf{M}}_{4}
\end{array}\right),
$$

with $\overline{\mathbf{M}}_{4}=\lim _{\lambda \rightarrow \infty} \mathbf{M}_{4}(\lambda)$, and we have

$$
\left|\lim _{\lambda \rightarrow \infty} \mathbf{K}(\lambda)\right|=\left|\mathbf{0} \overline{\mathbf{M}}_{4}-\left(-\mathbf{I}_{n}\right)^{2}\right|=-1^{n} .
$$

Next we note that

$$
\begin{gathered}
A^{1, i}(0)=\int_{0}^{\infty} \pi^{i}\left(a, \mathbf{P}_{*}\right) d a=\frac{P_{*}^{i}}{p_{*}^{i}(0)}, \quad i=1, \ldots, n, \\
A^{2, i}(0)=R^{i}\left(\mathbf{P}_{*}\right)=1, \quad i=1, \ldots, n, \\
A_{j}^{i}(0)=-p_{*}^{i}(0) \int_{0}^{\infty} \pi^{i}\left(a, \mathbf{P}_{*}\right) \int_{0}^{a} \mu_{P_{j}}^{i}\left(s, \mathbf{P}_{*}\right) d s d a, \quad i, j=1, \ldots, n, \\
\bar{A}_{j}^{i}(0)=\left(\frac{P_{*}^{i}}{\int_{0}^{\infty} \pi^{i}\left(a, \mathbf{P}_{*}\right) d a}\right) R_{P_{j}}^{i}\left(\mathbf{P}_{*}\right), \quad i, j=1, \ldots, n,
\end{gathered}
$$

which yields

$$
|\mathbf{K}(0)|=\left|\left(P_{*}^{i} R_{P_{j}}^{i}\left(\mathbf{P}_{*}\right)\right)_{(i, j)}\right| .
$$

Theorem 2.3. The stationary solution $\left(p_{*}^{1}(a), \ldots, p_{*}^{n}(a)\right)$ is unstable if

$$
\operatorname{sign}\left|\left(P_{*}^{i} R_{P_{j}}^{i}\left(\mathbf{P}_{*}\right)\right)_{(i, j)}\right|=\operatorname{sign}\left|\left(R_{P_{j}}^{i}\left(\mathbf{P}_{*}\right)\right)_{(i, j)}\right| \neq \operatorname{sign}\left(-1^{n}\right) .
$$

Proof. Theorem 2.2 and the continuity of $|\mathbf{K}(\lambda)|$ implies the existence of a positive solution of $|\mathbf{K}(\lambda)|=0$.

As an easy consequence of Theorem 2.3 in the case of $n=2$, we have the following.

Remark 2.4. Two-species competitive $\left(R_{P_{2}}^{1}, R_{P_{1}}^{2}<0\right)$ or cooperative $\left(R_{P_{2}}^{1}, R_{P_{1}}^{2}>0\right)$ systems where the interspecific interaction is stronger than the intraspecific interaction $\left(R_{P_{1}}^{1} R_{P_{2}}^{2}<\right.$ $R_{P_{2}}^{1} R_{P_{1}}^{2}$ ) do not admit stable positive steady states. 
6 Stability of an age-structured multispecies model

Next we note that we have $\mathbf{M}_{1}(\lambda) \mathbf{M}_{3}(\lambda)=\mathbf{M}_{3}(\lambda) \mathbf{M}_{1}(\lambda)$ in general; thus we can rewrite $|\mathbf{K}(\lambda)|$ as a determinant of an $n$-by- $n$ matrix:

$$
|\mathbf{K}(\lambda)|=\left|\left(\mathbf{M}_{1}(\lambda) \mathbf{M}_{4}(\lambda)-\mathbf{M}_{3}(\lambda) \mathbf{M}_{2}(\lambda)\right)\right|=\left|\left(\mathbf{K}(\lambda)_{(i, j)}\right)\right|
$$

where $\mathbf{K}(\lambda)_{(i, j)}$ is denoting the $i j$ th entry of the matrix $\mathbf{K}(\lambda)$ and

$$
\mathbf{K}(\lambda)_{(i \cdot j)}= \begin{cases}A^{1, i}(\lambda) \bar{A}_{j}^{i}(\lambda)-\left(A^{2, i}(\lambda)-1\right) A_{j}^{i}(\lambda) & \text { if } i \neq j, i, j=1, \ldots, n, \\ A^{1, i}(\lambda) \bar{A}_{j}^{i}(\lambda)-\left(A^{2, i}(\lambda)-1\right)\left(A_{j}^{i}(\lambda)-1\right) & \text { if } i=j, i=1, \ldots, n\end{cases}
$$

Observe that in the diagonal of $\mathbf{K}(\lambda)$ (i.e., $\left.\mathbf{K}(\lambda)_{(i, i)}, i=1, \ldots, n\right)$ we have the exact copies of characteristic functions of the one-dimensional age-structured problem as in [5].

According to [13], the linear system (2.2) is governed by a positive semigroup if

$$
\begin{gathered}
\sum_{j=1}^{n} P_{*}^{j} \mu_{P_{j}}^{i}\left(a, \mathbf{P}_{*}\right) \leq 0, \quad i=1, \ldots, n, a \in[0, \infty), \\
\beta^{i}\left(a, \mathbf{P}_{*}\right)+\sum_{j=1}^{n} P_{*}^{j} \beta_{P_{j}}^{i}\left(a, \mathbf{P}_{*}\right) \geq 0, \quad i=1, \ldots, n, a \in[0, \infty) .
\end{gathered}
$$

Remark 2.5. The positivity of the governing linear semigroup assure the existence of a dominant real root of the characteristic function. As we pointed out in [8] obtaining stability results is much harder than instability ones because we need to impose the technical and rather restrictive positivity conditions to stay in the framework of real calculus.

Consider the system (1.1) with the following vital rates:

$$
\begin{gathered}
\beta^{1}\left(P^{1}, \ldots, P^{n}\right), \quad \mu^{1}\left(P^{1}, \ldots, P^{n}\right), \ldots, \beta^{n-1}\left(P^{n-1}, P^{n}\right), \\
\mu^{n-1}\left(P^{n-1}, P^{n}\right), \quad \beta^{n}\left(P_{n}\right), \quad \mu^{n}\left(P_{n}\right),
\end{gathered}
$$

which can represent a food chain, for instance.

THeOREM 2.6. The stationary solution $\left(p_{*}^{1}(a), \ldots, p_{*}^{n}(a)\right)$ is asymptotically stable if

$$
\begin{gathered}
\mu_{P_{i}}^{i}\left(\cdot, \mathbf{P}_{*}\right)=0, \quad \mu_{P_{j}}^{i}\left(\cdot, \mathbf{P}_{*}\right)<0, \quad j>i=1, \ldots, n, \\
-\frac{\beta^{i}\left(\cdot, \mathbf{P}_{*}\right)}{P_{*}^{i}} \leq \beta_{P_{i}}^{i}\left(\cdot, \mathbf{P}_{*}\right)<0, \quad \beta_{P_{j}}^{i}\left(\cdot, \mathbf{P}_{*}\right)>0, \quad j>i=1, \ldots, n .
\end{gathered}
$$

Proof. By (2.25) we have $\bar{A}_{j}^{i}(\lambda)=A_{j}^{i}(\lambda)=0$ for $j<i=1, \ldots, n$ which means that $\mathbf{K}(\lambda)$ is an upper triangular matrix (by $(2.22)$ ), that is,

$$
|\mathbf{K}(\lambda)|=\prod_{i=1}^{n} \mathbf{K}(\lambda)_{(i, i)}=: \prod_{i=1}^{n} \mathbf{K}^{i}(\lambda) .
$$

First observe that $K^{i}(0)=P_{*}^{i} R_{P_{i}}^{i}\left(\mathbf{P}_{*}\right)<0, i=1, \ldots, n$, by $(2.26)-(2.27)$ that is $\operatorname{sign}(|\mathbf{K}(0)|)=$ $-1^{n}$. 
Now we want to show that $\mathbf{K}^{i}(\lambda), i=1, \ldots, n$, is monotone for $\lambda>0$,

$$
\begin{aligned}
\mathbf{K}^{i}(\lambda)^{\prime}= & A^{1, i}(\lambda)^{\prime} \bar{A}_{i}^{i}(\lambda)+A^{2, i}(\lambda)^{\prime} \\
= & -p_{*}^{i}(0) \int_{0}^{\infty} a e^{-\lambda a} \pi^{i}\left(a, \mathbf{P}_{*}\right) d a \int_{0}^{\infty} \beta_{P_{i}}^{i}\left(a, \mathbf{P}_{*}\right) \pi\left(a, \mathbf{P}_{*}\right) d a \\
& -\int_{0}^{\infty} a e^{\lambda a} \beta^{i}\left(a, \mathbf{P}_{*}\right) \pi^{i}\left(a, \mathbf{P}_{*}\right) d a \\
= & \int_{0}^{\infty} a e^{-\lambda a} \pi^{i}\left(a, \mathbf{P}_{*}\right)\left(p_{*}^{i}(0) \int_{0}^{\infty}\left(-\beta_{P_{i}}^{i}\left(s, \mathbf{P}_{*}\right)\right) \pi^{i}\left(s, \mathbf{P}_{*}\right) d s-\beta^{i}\left(a, \mathbf{P}_{*}\right)\right) d a \\
= & \int_{0}^{\infty} a e^{-\lambda a} \pi^{i}\left(a, \mathbf{P}_{*}\right)\left(\int_{0}^{\infty}\left(-\beta_{P_{i}}^{i}\left(s, \mathbf{P}_{*}\right)\right) p_{*}^{i}(s) d s-\beta^{i}\left(a, \mathbf{P}_{*}\right)\right) d a \leq 0 .
\end{aligned}
$$

The last inequality follows from

$$
\beta^{i}\left(\cdot, \mathbf{P}_{*}\right) \geq-P_{*}^{i} \beta_{P_{i}}^{i}\left(\cdot, \mathbf{P}_{*}\right) \geq-\int_{0}^{\infty} \beta_{P_{i}}^{i}\left(s, \mathbf{P}_{*}\right) p_{*}^{i}(s) d s
$$

by (2.27). Observe that by (2.26)-(2.27) all the positivity conditions (2.23)-(2.24) are satisfied thus we just need to consider the characteristic function along the reals. Applying Theorem 2.2 one has that the characteristic function (2.21) does not have a nonnegative real root, since $\operatorname{sign}(|\mathbf{K}(0)|)=\lim _{\lambda \rightarrow \infty}|\mathbf{K}(\lambda)|=-1^{n}$, thus applying Theorem 2.1 the stability of the stationary solution follows.

Remark 2.7. In the case of vital rates as in $(2.25) R_{P_{i}}^{i}\left(\mathbf{P}_{*}\right)>0$ for any $i \in\{1, \ldots, n\}$ implies instability.

\section{Acknowledgments}

I am thankful to the anonymous referees for helpful comments and suggestions.

\section{References}

[1] O. Diekmann, M. Gyllenberg, H. Huang, M. Kirkilionis, J. A. J. Metz, and H. R. Thieme, On the formulation and analysis of general deterministic structured population models. II. Nonlinear theory, Journal of Mathematical Biology 43 (2001), no. 2, 157-189.

[2] O. Diekmann, M. Gyllenberg, and J. A. J. Metz, Physiologically structured population models: toward a general mathematical theory, preprint.

[3] O. Diekmann, M. Gyllenberg, J. A. J. Metz, and H. R. Thieme, On the formulation and analysis of general deterministic structured population models. I. Linear theory, Journal of Mathematical Biology 36 (1998), no. 4, 349-388.

[4] K.-J. Engel and R. Nagel, One-Parameter Semigroups for Linear Evolution Equations, Graduate Texts in Mathematics, vol. 194, Springer, New York, 2000.

[5] M. Farkas, On the stability of stationary age distributions, Applied Mathematics and Computation 131 (2002), no. 1, 107-123.

[6] J. Z. Farkas, Stability conditions for the non-linear McKendrick equations, Applied Mathematics and Computation 156 (2004), no. 3, 771-777.

[7] Stability conditions for a non-linear size-structured model, Nonlinear Analysis. Real World Applications 6 (2005), no. 5, 962-969. 


\section{$8 \quad$ Stability of an age-structured multispecies model}

[8] J. Z. Farkas and T. Hagen, Stability and regularity results for a size-structured population model, to appear in Journal of Mathematical Analysis and Applications.

[9] M. E. Gurtin and R. C. MacCamy, Non-linear age-dependent population dynamics, Archive for Rational Mechanics and Analysis 54 (1974), 281-300.

[10] M. Iannelli, Mathematical Theory of Age-Structured Population Dynamics, Giardini Editori, Pisa, 1994.

[11] N. Kato, A principle of linearized stability for nonlinear evolution equations, Transactions of the American Mathematical Society 347 (1995), no. 8, 2851-2868.

[12] J. A. J. Metz and O. Diekmann, The Dynamics of Physiologically Structured Populations, Lecture Notes in Biomathematics, vol. 68, Springer, Berlin, 1986.

[13] J. Prüß, Stability analysis for equilibria in age-specific population dynamics, Nonlinear Analysis. Theory, Methods \& Applications 7 (1983), no. 12, 1291-1313.

[14] G. F. Webb, Theory of Nonlinear Age-Dependent Population Dynamics, Monographs and Textbooks in Pure and Applied Mathematics, vol. 89, Marcel Dekker, New York, 1985.

Jozsef Z. Farkas: Department of Mathematical Sciences, The University of Memphis, Memphis, TN 38152, USA

E-mail address: jzfarkas@memphis.edu 


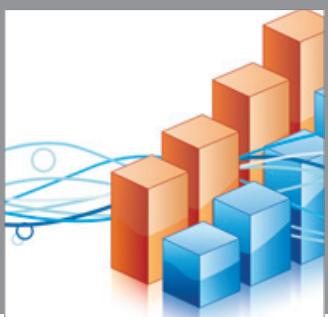

Advances in

Operations Research

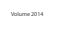

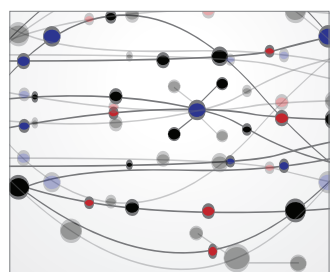

\section{The Scientific} World Journal
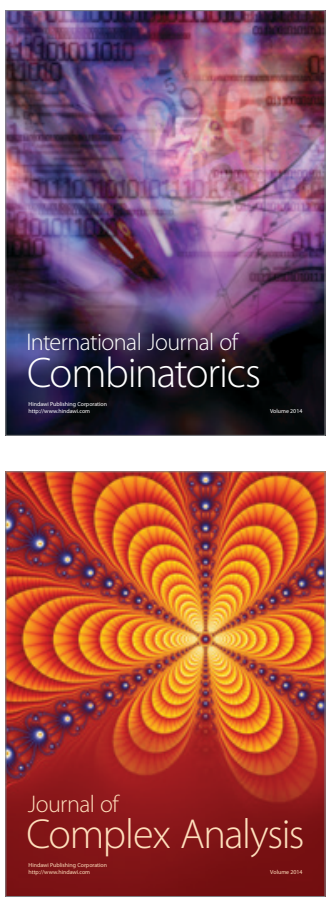

International Journal of

Mathematics and

Mathematical

Sciences
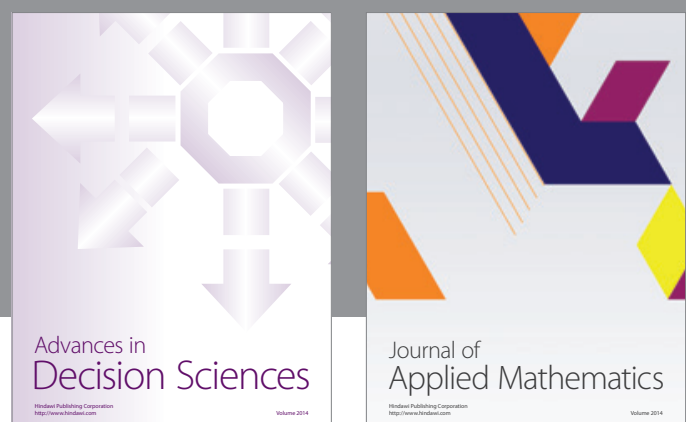

Journal of

Applied Mathematics
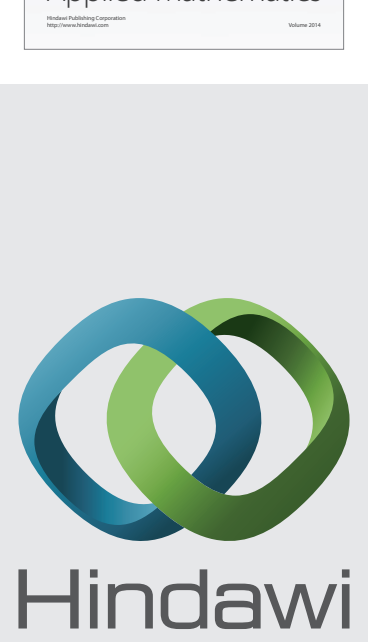

Submit your manuscripts at http://www.hindawi.com
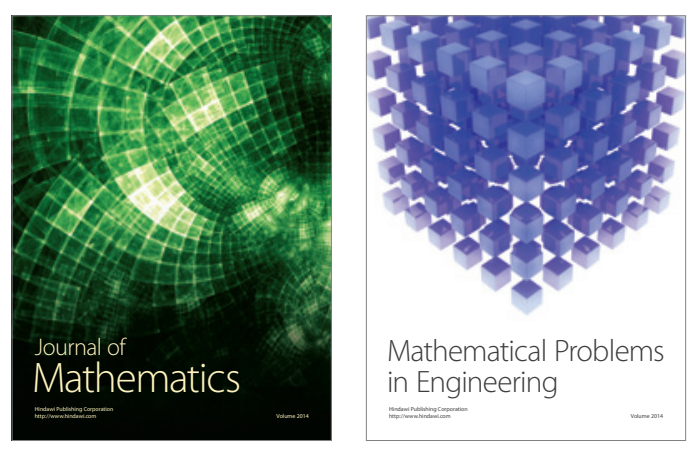

Mathematical Problems in Engineering
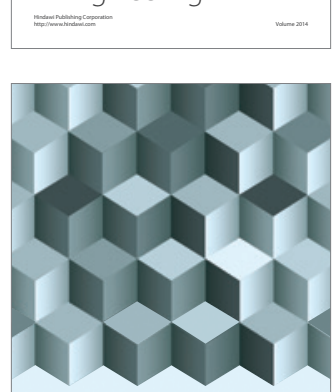

Journal of

Function Spaces
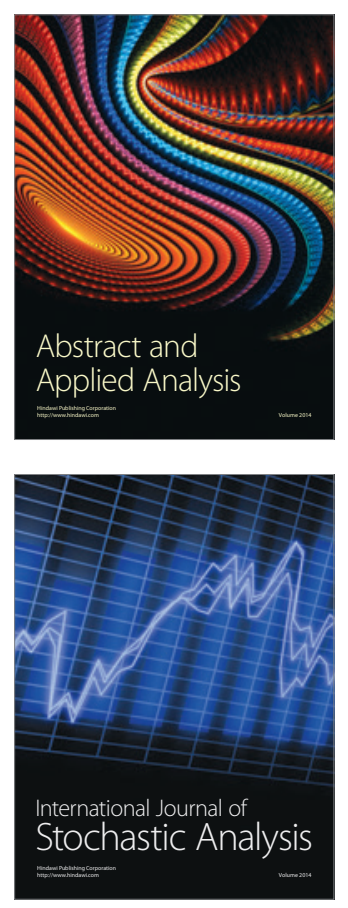

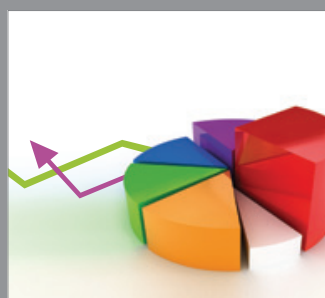

ournal of

Probability and Statistics

Promensencen
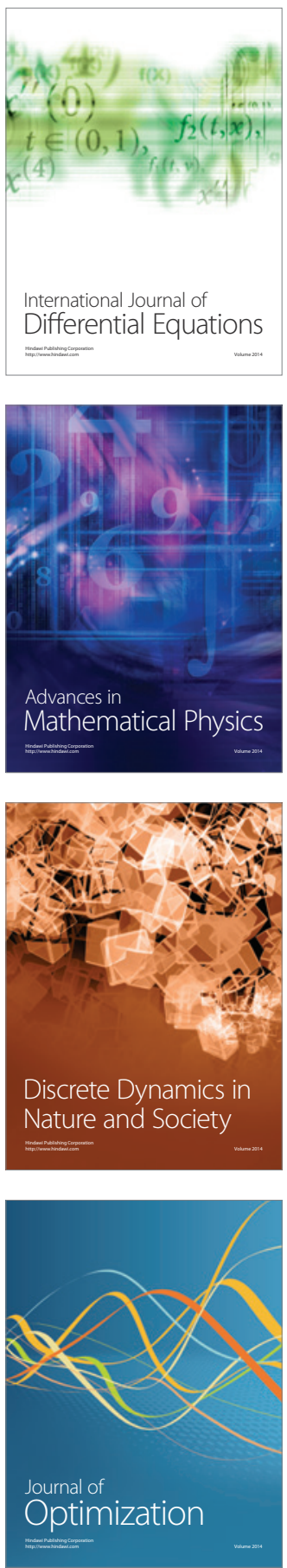